\title{
Protocol for a randomized, placebo-controlled, double-blind clinical trial investigating sacral neuromodulation for neurogenic lower urinary tract dysfunction
}

Stephanie C Knüpfer ${ }^{1}$, Martina D Liechti ${ }^{1}$, Livio Mordasini ${ }^{2}$, Dominik Abt ${ }^{2}$, Daniel S Engeler ${ }^{2}$, Jens Wöllner ${ }^{3}$, Jürgen Pannek ${ }^{3}$, Bernhard Kiss ${ }^{4}$, Fiona C Burkhard ${ }^{4}$, Marc P Schneider ${ }^{1}$, Elena Miramontes ${ }^{1}$, Alfons G Kessels ${ }^{5}$, Lucas M Bachmann ${ }^{6}$ and Thomas M Kessler ${ }^{1 *}$

\begin{abstract}
Background: Sacral neuromodulation has become a well-established and widely accepted treatment for refractory non-neurogenic lower urinary tract dysfunction, but its value in patients with a neurological cause is unclear. Although there is evidence indicating that sacral neuromodulation may be effective and safe for treating neurogenic lower urinary tract dysfunction, the number of investigated patients is low and there is a lack of randomized controlled trials.
\end{abstract}

Methods and design: This study is a prospective, randomized, placebo-controlled, double-blind multicenter trial including 4 sacral neuromodulation referral centers in Switzerland. Patients with refractory neurogenic lower urinary tract dysfunction are enrolled. After minimally invasive bilateral tined lead placement into the sacral foramina S3 and/or S4, patients undergo prolonged sacral neuromodulation testing for 3-6 weeks. In case of successful (defined as improvement of at least 50\% in key bladder diary variables (i.e. number of voids and/or number of leakages, post void residual) compared to baseline values) prolonged sacral neuromodulation testing, the neuromodulator is implanted in the upper buttock. After a 2 months post-implantation phase when the neuromodulator is turned ON to optimize the effectiveness of neuromodulation using sub-sensory threshold stimulation, the patients are randomized in a 1:1 allocation in sacral neuromodulation ON or OFF. At the end of the 2 months double-blind sacral neuromodulation phase, the patients have a neuro-urological re-evaluation, unblinding takes place, and the neuromodulator is turned $\mathrm{ON}$ in all patients. The primary outcome measure is success of sacral neuromodulation, secondary outcome measures are adverse events, urodynamic parameters, questionnaires, and costs of sacral neuromodulation.

Discussion: It is of utmost importance to know whether the minimally invasive and completely reversible sacral neuromodulation would be a valuable treatment option for patients with refractory neurogenic lower urinary tract dysfunction. If this type of treatment is effective in the neurological population, it would revolutionize the management of neurogenic lower urinary tract dysfunction.

Trial registration: Trial registration number: www.clinicaltrials.gov; Identifier: NCT02165774.

Keywords: Urinary bladder, Neurogenic lower urinary tract dysfunction, Sacral neuromodulation, Randomized, Placebo-controlled, Double-blind trial

\footnotetext{
* Correspondence: tkessler@gmx.ch

${ }^{1}$ Neuro-Urology, Spinal Cord Injury Center \& Research, University of Zürich,

Balgrist University Hospital, Forchstrasse 340, 8008 Zürich, Switzerland

Full list of author information is available at the end of the article
} 


\section{Background}

Neurogenic lower urinary tract dysfunction (LUTD) is highly prevalent and affects the lives of millions of people worldwide. It has a major impact on quality of life and, besides the debilitating manifestations for patients, it also imposes a substantial economic burden for every healthcare system. Neurogenic LUTD is a challenge because all available treatment modalities (i.e. conservative, minimally invasive and surgical therapies) may either fail or be invasive causing considerable complications and/or side effects.

Sacral neuromodulation (SNM) [1] has become a wellestablished and widely accepted treatment for patients with refractory LUTD such as non-obstructive chronic urinary retention, urgency frequency syndrome, and urgency incontinence [2-6] and it has been incorporated into the guidelines of the European Association of Urology (EAU) (www.uroweb.org), the International Consultation on Incontinence (ICI) [7], and the National Institute for Health and Clinical Excellence (NICE) (www.nice.org.uk). Originally, SNM was not considered an option for neurogenic LUTD but some studies suggested that it is also effective in neurological patients $[3,8]$. Considering that SNM is minimally invasive and completely reversible, it is of great interest whether this is a valuable treatment option for patients with neurogenic LUTD. In a recent systematic review and meta-analysis [9], we found that there is evidence indicating that SNM may be effective and safe for the treatment of this group of patients. However, the number of investigated patients is low with high between-study heterogeneity and there is a lack of randomized controlled trials [9].

We therefore designed a prospective, randomized, placebo-controlled, double-blind multicenter clinical trial to assess the efficacy and safety of SNM for treating patients with neurogenic LUTD. The study hypothesis is that in patients with refractory neurogenic LUTD, SNM leads to an at least 35\% increase in success rate as compared to placebo (i.e. sham) stimulation within 2 months, i.e. SNM is considerably more effective than placebo (i.e. sham) stimulation.

\section{Methods and design Study design}

This study is a prospective, randomized, placebo-controlled, double-blind multicenter trial including 4 SNM referral centers in Switzerland: Neuro-Urology, Spinal Cord Injury Center \& Research, University of Zürich, Balgrist University Hospital, Zürich; Department of Urology, Cantonal Hospital St. Gallen, St. Gallen; Neuro-Urology, Swiss Paraplegic Center Nottwil, Nottwil; Department of Urology, University of Bern, Bern.

In case of successful prolonged SNM, the neuromodulator is implanted and patients are randomized using a computer program considering 3 strata according to the neuro-urological diagnosis, i.e. a) urgency frequency syndrome and/or urgency incontinence, b) chronic urinary retention, and c) combination of urgency frequency syndrome and/or urgency incontinence and chronic urinary retention. After a 2 months SNM optimization phase following neuromodulator implantation, the neuromodulator is turned ON or OFF in a 1:1 allocation by an investigator not involved in the assessment of the clinical outcome.

The Figure 1 gives an overview of the procedures that the patients will undergo during the course of the study.

\section{Study population and recruitment}

According to the inclusion and exclusion criteria (Table 1), we will investigate patients with refractory neurogenic LUTD. The following variables will be considered: gender, age, neurological disease, duration of neurological disease, previous treatment, bladder diary variables, urinalysis, urethro-cystoscopy, bladder washing cytology, urodynamic investigations, and questionnaires, i.e. Female Sexual Function Index (FSFI) [10]/International Index of Erectile Function (IIEF) [11] and Qualiveen [12].

\section{Determination of sample size}

We are planning a study of independent cases and controls with 1 control per case. Prior data indicate that the (spontaneous) success rate among controls is 0.15 . If the true success rate for experimental subjects (SNM ON) is at least 0.5 , we will need to study 27 experimental subjects (SNM ON) and 27 control subjects (SNM OFF) to be able to reject the null hypothesis that the failure rates for experimental (SNM ON) and control subjects (SNM OFF) are equal with probability (power) 0.8 . The type I error probability associated with this test of this null hypothesis is 0.05 . Taking into account potential dropouts, we will include 30 patients per group.

\section{Study location and partners}

- Neuro-Urology, Spinal Cord Injury Center \& Research, University of Zürich, Balgrist University Hospital, Zürich, Switzerland

- Department of Urology, Cantonal Hospital St. Gallen, St. Gallen, Switzerland

- Neuro-Urology, Swiss Paraplegic Center, Nottwil, Switzerland

- Department of Urology, University of Bern, Switzerland

- Department of Clinical Epidemiology and Medical Technology Assessment, Maastricht University Medical Center, Maastricht, The Netherlands

- Medignition Inc., Research Consultants, Zug, Switzerland 


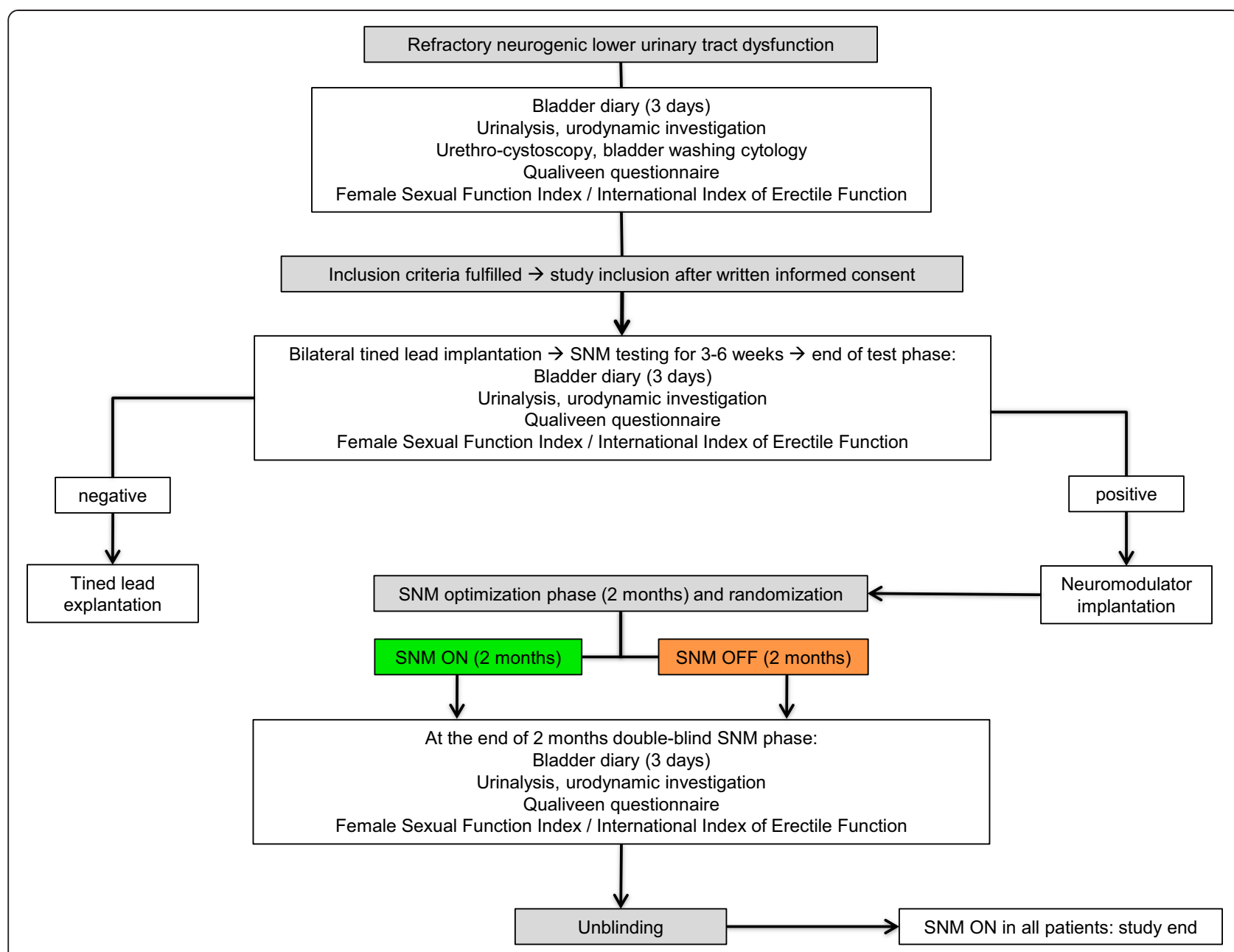

Figure 1 Flowchart of the sacral neuromodulation (SNM) trial. SNM: sacral neuromodulation.

\section{Investigations}

In case the patients with refractory neurogenic LUTD fulfill the study inclusion criteria following neuro-urological evaluation (bladder diary for at least 3 days, urinalysis, urodynamic investigation, urethro-cystoscopy and bladder washing cystology, Qualiveen questionnaire [12], FSFI [10]/IIEF [11], they are included after providing written informed consent. The Figure 1 gives an overview of the procedures that the patients will undergo during the course of the study. After minimally invasive bilateral

Table 1 Inclusion and exclusion criteria for patients with refractory neurogenic lower urinary tract dysfunction (LUTD)

\section{Inclusion criteria}

- Age $>18$ years

- Refractory neurogenic LUTD

- Urgency frequency syndrome and/or urgency incontinence refractory to antimuscarinics (pharmacotherapy for at least 4 weeks with at least 2 antimuscarinics)

- Chronic urinary retention refractory to alpha-blocker (pharmacotherapy with an alpha-blocker for at least 4 weeks)

- Combination of urgency frequency syndrome and/or urgency incontinence refractory to antimuscarinics (pharmacotherapy for at least 4 weeks with at least 2 antimuscarinics) and chronic urinary retention refractory to alpha-blocker (pharmacotherapy with an alpha-blocker for at least 4 weeks)

- Written informed consent
Exclusion criteria

- Age $<18$ years

- Non-neurogenic LUTD

- Botulinum toxin injections into the detrusor and/or urethral sphincter in the last 6 months

- Pregnancy or breast feeding

- Individuals especially in need of protection (according to Research with Human Subjects published by the Swiss Academy of Medical Sciences (www.samw.ch/en/News/News.html))

- No written informed consent 
tined lead placement into the sacral foramina S3 and/or S4 (stage one), patients undergo prolonged SNM testing for 3-6 weeks completing a bladder diary to assess the response to treatment. In accordance with the literature $[5,6]$, an improvement of at least $50 \%$ in the key bladder diary variables (i.e. number of voids and/or number of leakages, post void residual) compared to the baseline values is considered a positive test and an indication for neuromodulator implantation. At the end of the test phase, the patients have a neuro-urological re-evaluation (bladder diary for at least 3 days, urinalysis, urodynamic investigation, Qualiveen questionnaire [12], FSFI [10]/IIEF [11]). In case of negative prolonged SNM testing, the tined leads are explanted. In case of successful prolonged SNM testing, the neuromodulator is generally implanted in the upper buttock (rarely in the anterior abdominal wall) (stage two). After neuromodulator implantation, each patient has a 2 months phase when the neuromodulator is turned ON using sub-sensory threshold stimulation (SNM optimization phase) to optimize the effectiveness of neuromodulation by determining the most effective stimulation parameters (choice of stimulation electrodes, intensity of stimulation) for each patient. At the end of the SNM optimization phase, patients are randomized in a double-blind parallel design to SNM ON or OFF. During an outpatient visit, neuromodulation parameters and bladder diary parameters are checked and the neuromodulator is turned ON or OFF by an investigator not involved in assessment of the clinical outcome. Considering that sub-sensory stimulation is used for SNM, the patients do not feel if the stimulation is ON or OFF. At the end of the 2 months doubleblind SNM phase, the patients have a neuro-urological re-evaluation (bladder diary for at least 3 days, urinalysis, urodynamic investigation, Qualiveen questionnaire [12], FSFI [10]/IIEF [11]). During this visit, unblinding takes place and the neuromodulator is turned $\mathrm{ON}$ in all patients.

\section{Safety}

The investigators will inform the patients, the study monitoring board, and the ethics committee if it becomes evident that the disadvantages of participation may be significantly greater than was foreseen in the research proposal. The study will be suspended pending further review by the study monitoring board, except insofar as suspension would jeopardize the patients' health. The investigators will take care that all patients are kept informed.

Adverse events will be assessed and categorized according to the National Cancer Institute Common Terminology Criteria for Adverse Events (CTCAE) version 4 in grade 1 to 5 (http://ctep.cancer.gov/protocolDevelopment/ electronic_applications/ctc.htm). All adverse events will be followed until they have abated, or until a stable situation has been reached. Depending on the event, follow-up may require additional tests or medical procedures as indicated, and/or referral to the general physician or a medical specialist.

In the case of withdrawal of consent to participate in the study, all possible efforts will be made to convince the patient to continue to have safety follow-up evaluations.

In the event one of the following situations arises among treated patients during the conduct of the study, the study will be temporarily suspended and a comprehensive safety review conducted evaluating if the study has to be terminated prematurely:

- Any death secondary to rapid unexpected progression of an underlying medical condition.

- Severe clinical or neurological deterioration in more than one subject.

- Any other serious adverse event determined by the study monitoring board to be a reason to suspend the study.

\section{Study outcome measures}

Primary: Success of SNM: Defined in accordance with the literature $[5,6]$ as improvement of at least $50 \%$ in the key bladder diary variables (i.e. number of voids and/or number of leakages, post void residual) compared to the baseline values (i.e. patients with urgency frequency syndrome and/or urgency incontinence: at least $50 \%$ decrease in number of voids and/or number of leakages; patients with chronic urinary retention: at least $50 \%$ decrease in post void residual; patients with a combination of urgency frequency syndrome and/or urgency incontinence and chronic urinary retention: at least $50 \%$ decrease in number of voids and/or number of leakages, and/or at least 50\% decrease in post void residual).

Secondary: A) Adverse events: Categorization according to the National Cancer Institute Common Terminology Criteria for Adverse Events (CTCAE) version 4 in grade 1 to 5 (http://ctep.cancer.gov/protocolDevelopment/ electronic_applications/ctc.htm).

B) Urodynamic parameters: cystometric capacity $(\mathrm{mL})$, compliance $\left(\mathrm{mL} / \mathrm{cmH}_{2} \mathrm{O}\right)$, detrusor overactivity (if yes: bladder volume $(\mathrm{mL})$ at detrusor overactivity, maximum detrusor pressure amplitude $\left(\mathrm{cmH}_{2} \mathrm{O}\right)$, detrusor leak point pressure $\left.\left(\mathrm{cmH}_{2} \mathrm{O}\right)\right)$, maximum detrusor pressure $\left(\mathrm{cmH}_{2} \mathrm{O}\right)$, detrusor pressure at maximum flow rate $\left(\mathrm{cmH}_{2} \mathrm{O}\right)$, maximum flow rate $(\mathrm{mL} / \mathrm{s})$, voided volume $(\mathrm{mL})$, post void residual $(\mathrm{mL})$, pelvic floor electromyographic activity (normal/detrusor sphincter dyssynergia).

C) Questionnaires, i.e. Qualiveen and FSFI/IIEF.

D) Costs of SNM. 


\section{Data analysis Statistics}

Interval scaled variates will be summarized with means and standard deviations (SD) or medians and interquartile ranges where appropriate. Dichotomous variates will be described as ratios and percentages.

\section{Univariate analysis}

T-tests will be used to compare means between groups and chi-squared tests to compare dichotomous variables.

\section{Multivariate analysis}

To adjust for unequal distribution of parameters at baseline, multivariate regression models, linear models in case of an interval scaled outcome and logistic regression in case of a dichotomous outcome will be performed.

\section{Ethics and dissemination}

This trial will be performed in accordance with the World Medical Association Declaration of Helsinki [13], the guidelines for Good Clinical Practice [14] and the guidelines of the Swiss Academy of Medical Sciences [15]. Handling of all personal data will strictly comply with the federal law of data protection in Switzerland [16]. The trial has been registred at clinicaltrials.gov (www. clinicaltrials.gov/ct2/show/NCT02165774).

\section{Discussion}

First-line treatment for neurogenic LUTD includes antimuscarinics and some form of catheterization if necessary, preferably intermittent self-catheterization [17]. However, the treatment effect is often unsatisfactory, so that other options have to be considered, including onabotulinumtoxinA injections into the detrusor [18] or more invasive procedures such as bladder augmentation or urinary diversions. Thus, it is of utmost importance to know whether the minimally invasive and completely reversible SNM, a well established and widely accepted therapy for refractory non-neurogenic LUTD, would be a valuable treatment option for patients with refractory neurogenic LUTD. In addition, SNM may enable voiding without intermittent catheterization, the standard technique for chronic neurogenic urinary retention today. As a significant number of patients cannot perform this technique due to the underlying neurological disorder, SNM may not only prevent major surgery but also life-long treatment with indwelling catheters, which are related to significant long-term complications.

Assessing efficacy and safety of SNM for neurogenic LUTD, it is essential to be aware of the fact that these patients usually have undergone multiple failed previous treatments. In the case that SNM is also effective in the neurological population, this would have major implications for daily practice and would completely revolutionize the management of neurogenic LUTD.

This trial is multidisciplinary and will significantly influence all involved disciplines, i.e. neuro-urology, urology, and neurology. Especially in neurology, this project will increase the awareness of LUTD in neurological disorders and the related effective treatment options including SNM.

\section{Ethics approval}

This study has been approved by the local ethics committees (Kantonale Ethikkommission Zürich KEK-ZHNr. 2011-0048, St. Gallen KEK-SG-Nr. 12/069, Luzern KEK-LU-Nr. 12047, Bern KEK-BE-Nr. 094/12).

\section{Abbreviations}

CTCAE: Common Terminology Criteria for Adverse Events; EAU: European Association of Urology; FSFI: Female Sexual Function Index; ICl: International Consultation on Incontinence; IIEF: International Index of Erectile Function; LUTD: Lower urinary tract dysfunction; NICE: National Institute for Health and Clinical Excellence; SNM: Sacral neuromodulation.

\section{Competing interests}

The authors declare that they have no competing interests.

\section{Authors' contributors}

TMK, DSE, AGK, and LMB created the study design. SCK and TMK drafted the manuscript. MDL, LM, DA, DSE, JW, JP, BK, FCB, MPS, EM, AGK, and LMB critically reviewed the manuscript. TMK obtained the funding of this study. All the authors read and approved the final manuscript.

\section{Acknowledgements}

The authors would like to acknowledge the Swiss National Science Foundation, Vontobel-Stiftung, Gottfried und Julia Bangerter-Rhyner-Stiftung, Dr. Urs Mühlebach, and Swiss Continence Foundation for financial support.

\section{Funding}

Swiss National Science Foundation, Vontobel-Stiftung, Gottfried und Julia Bangerter-Rhyner-Stiftung, Dr. Urs Mühlebach, and Swiss Continence Foundation.

\section{Author details}

${ }^{1}$ Neuro-Urology, Spinal Cord Injury Center \& Research, University of Zürich, Balgrist University Hospital, Forchstrasse 340, 8008 Zürich, Switzerland.

${ }^{2}$ Department of Urology, Cantonal Hospital St. Gallen, St. Gallen, Switzerland. ${ }^{3}$ Neuro-Urology, Swiss Paraplegic Center, Nottwil, Switzerland. ${ }^{4}$ Department of Urology, University of Bern, Bern, Switzerland. ${ }^{5}$ Department of Clinical Epidemiology and Medical Technology Assessment, Maastricht University Medical Center, Maastricht, The Netherlands. ${ }^{6}$ Medignition Inc., Research Consultants, Zug, Switzerland.

Received: 15 July 2014 Accepted: 9 August 2014 Published: 13 August 2014

\section{References}

1. Wöllner J, Hampel C, Kessler TM: Surgery illustrated - surgical atlas sacral neuromodulation. BJU Int 2012, 110(1):146-159.

2. Brazzelli M, Murray A, Fraser C: Efficacy and safety of sacral nerve stimulation for urinary urge incontinence: a systematic review. J Urol 2006, 175(3 Pt 1):835-841.

3. Chartier-Kastler EJ, Ruud Bosch JL, Perrigot M, Chancellor MB, Richard F, Denys P: Long-term results of sacral nerve stimulation (S3) for the treatment of neurogenic refractory urge incontinence related to detrusor hyperreflexia. J Urol 2000, 164(5):1476-1480.

4. Herbison GP, Arnold EP: Sacral neuromodulation with implanted devices for urinary storage and voiding dysfunction in adults. Cochrane Database Syst Rev 2009, 2:CD004202. 
5. Kessler TM, Buchser E, Meyer S, Engeler DS, Al-Khodairy AW, Bersch U, Iselin CE, Roche B, Schmid DM, Schurch B, Zrehen S, Burkhard FC: Sacral neuromodulation for refractory lower urinary tract dysfunction: results of a nationwide registry in Switzerland. Eur Urol 2007, 51(5):1357-1363.

6. van Kerrebroeck PE, van Voskuilen AC, Heesakkers JP, Lycklama a Nijholt AA, Siegel S, Jonas U, Fowler CJ, Fall M, Gajewski JB, Hassouna MM, Cappellano F, Elhilali MM, Milam DF, Das AK, Dijkema HE, van den Hombergh U: Results of sacral neuromodulation therapy for urinary voiding dysfunction: outcomes of a prospective, worldwide clinical study. J Urol 2007, 178(5):2029-2034.

7. Abrams P, Andersson KE, Birder L, Brubaker L, Cardozo L, Chapple C, Cottenden A, Davila W, de Ridder D, Dmochowski R, Drake M, Dubeau C, Fry C, Hanno P, Smith JH, Herschorn S, Hosker G, Kelleher C, Koelbl H, Khoury S, Madoff R, Milsom I, Moore K, Newman D, Nitti V, Norton C, Nygaard I, Payne C, Smith A, Staskin D, et al: Fourth International Consultation on Incontinence Recommendations of the International Scientific Committee: evaluation and treatment of urinary incontinence, pelvic organ prolapse, and fecal incontinence. Neurourol Urodyn 2010, 29(1):213-240

8. Wallace PA, Lane FL, Noblett KL: Sacral nerve neuromodulation in patients with underlying neurologic disease. Am J Obstet Gynecol 2007, 197(1):96. e91-95.

9. Kessler TM, La Framboise D, Trelle S, Fowler CJ, Kiss G, Pannek J, Schurch B, Sievert KD, Engeler DS: Sacral neuromodulation for neurogenic lower urinary tract dysfunction: systematic review and meta-analysis. Eur Urol 2010, 58(6):865-874

10. Kriston L, Gunzler C, Rohde A, Berner MM: Is one question enough to detect female sexual dysfunctions? A diagnostic accuracy study in 6,194 women. J Sex Med 2010, 7(5):1831-1841.

11. Kriston L, Gunzler C, Harms A, Berner M: Confirmatory factor analysis of the German version of the international index of erectile function (IIEF): a comparison of four models. J Sex Med 2008, 5(1):92-99.

12. Costa P, Perrouin-Verbe B, Colvez A, Didier J, Marquis P, Marrel A, Amarenco G, Espirac B, Leriche A: Quality of life in spinal cord injury patients with urinary difficulties. Development and validation of qualiveen. Eur Urol 2001, 39(1):107-113.

13. Association WM: World Medical Association Declaration of Helsinki: ethical principles for medical research involving human subjects. JAMA 2013, 310(20):2191-2194.

14. International conference on harmonisation: Good clinical practice guideline; 1996 [http//www.ich.org/products/guidelines/efficacy/article/efficacy-guidelines.html]

15. Swiss Academy of Medical Sciences: Guideline - Concerning scientific research involving human beings; 2009 [www.samw.ch/dms/de/Publikationen/ Leitfaden/d_Forschung-mit-Menschen.pdf]

16. The Federal Authorities of the Swiss Confederation: Bundesgesetz über den Datenschutz (DSG) vom 19. Juni 1992; 1992 [http://www.admin.ch/ch/d/sr/2/ 235.1.de.pdf]

17. Pannek J, Blok B, Castro-Diaz D, Del Popolo G, Groen J, Karsenty G, Kessler TM, Kramer G, Stöhrer M: EAU guidelines on neuro-urology; 2014 [http://www. uroweb.org/gls/pdf/21\%20Neuro-Urology_LR.pdf]

18. Wöllner J, Kessler TM: Botulinum toxin injections into the detrusor. BJU Int 2011, 108(9):1528-1537.

doi:10.1186/1471-2490-14-65

Cite this article as: Knüpfer et al.: Protocol for a randomized,

placebo-controlled, double-blind clinical trial investigating sacral neuromodulation for neurogenic lower urinary tract dysfunction. $B M C$ Urology 2014 14:65

\section{Submit your next manuscript to BioMed Central and take full advantage of:}

- Convenient online submission

- Thorough peer review

- No space constraints or color figure charges

- Immediate publication on acceptance

- Inclusion in PubMed, CAS, Scopus and Google Scholar

- Research which is freely available for redistribution 